\title{
Zum deutschen Diokletiansbild im 19. Jahrhundert
}

\section{Hartmut Leppin}

Diokletian und Konstantin gehören, so sollte man meinen, in der historischen Tradition aufs engste zusammen: Hier der Christenverfolger, da der Christenfreund; diese Differenz mußte eine christlich geprägte Geschichtsschreibung zunächst beherrschen. Allerdings gab es andere Taten Diokletians, die um so schwerer ins Gewicht fielen, je mehr die Geschichtsschreibung bereit war, säkulare Kategorien anzuwenden: Er hatte das römische Reich stabilisiert und neu geordnet. Auch sonst vermochte seine Persönlichkeit den Eindruck sittlicher Integrität zu erwecken; dafür stand etwa sein Rücktritt vom Herrscheramt aus freien Stücken. Aufgrund solcher Beobachtungen und in dem Willen, das christliche Geschichtsbild umzukehren, hatten zumal Voltaire (1694-1778) und Edward Gibbon (1737-1794) sehr wohlwollende Bewertungen Diokletians ausgesprochen. ${ }^{1}$

In der deutschsprachigen Forschung des 19. Jahrhunderts spielt Diokletian, mustert man lediglich die Titel von Monographien durch, keine große Rolle. Das wenige, was erschien, stammt nicht von Gelehrten aus der ersten Reihe. Allerdings ist dieser erste Eindruck schief: Denn in verschiedenen Werken nimmt die Behandlung Diokletians breiten Raum ein, auch wenn sein Name im Titel fehlt. Einige Varianten der Einschätzung Diokletians werde ich zunächst anhand der Arbeit von vier Forschern aus der zweiten Hälfte des 19. Jahrhunderts vorführen, die in Hinblick auf ihr religiöses Bekenntnis unterschiedliche Haltungen einnehmen. Zwei stehen dem zeitgenössischen Christentum fern: Jacob Burckhardt und Theodor Mommsen; zwei sind bekennende Christen: Albrecht Vogel und Theodor Preuß; im Anschluß daran soll die Sonderposition des Agnostikers Otto Seeck zur Sprache kommen. ${ }^{2}$ Eine erschöpfende Behandlung der einschlägigen Partien ist im Rahmen dieses Beitrags nicht möglich. Es soll vielmehr, nachdem der Rahmen abgesteckt ist, ihre Einschätzung der Chri-

1 Vgl. nur KAEGI 1956, 381f; s. auch exemplarisch für eine differenzierende Einschätzung im deutschsprachigen Raum SCHLOSSER 1846, $411 \mathrm{ff}$., der Diokletian einerseits Bedachtsamkeit zuschreibt, der andererseits die Christenverfolgung als ein Ereignis sieht, das im Einklang mit Diokletians auch sonst aufflackernder Grausamkeit stehe.

2 Ausgespart bleiben aus pragmatischen Gründen Arbeiten wie MADER 1847 und HUNZIKER 1868. 
stenverfolgung näher erläutert werden. Der Schwerpunkt liegt klar auf der Arbeit Burckhardts, dessen Werk zum Bezugspunkt der späteren Forscher geworden ist. ${ }^{3}$ Eine umfassende Untersuchung zum Diokletiansbild im 18./19. Jahrhundert wäre gewiß lohnend, mehr als ein Anstoß kann die vorliegende Studie nicht sein. ${ }^{4}$

Jacob Burckhardts auf 1853 datierte, aber bereits Ende 1852 erschienene „Zeit Constantins des Großen“" widmet sich in einem großen Umfang Diokletian. ${ }^{5}$ Das berühmte Panorama der Provinzen schildert über weite Strecken die Zustände unter seiner Herrschaft; zudem ist ein eigenes Kapitel diesem Kaiser gewidmet, dessen Thronbesteigung den zeitlichen Beginn der Darstellung markiert (3). Die nachhaltigste historiographische Leistung Burckhardts bildet in dieser Partie die Entwicklung der Vorstellung einer diokletianischen Herrschaftsordnung, die von ihm in der Kapitelüberschrift als das "System seiner Adoptionen“ bezeichnet wird; es ist das, was heute als Tetrarchie bezeichnet wird, mit einem Begriff, der offenbar erst in den 80er Jahren des 19. Jahrhunderts von einem eher randständigen Gelehrten, Hermann Schiller, eingeführt wurde. ${ }^{6}$

Diese diokletianische Regierungsform handelt der Baseler detailliert ab und unterstreicht, daß sie gezielt aufgebaut worden sei; in der zweiten Auflage betont er die Geschlossenheit des Systems noch stärker als in der ersten. Was hier geschaffen wird, erhält vom Baseler höchstes Lob: Es sei gelungen, Usurpationen zu verhindern und die Arbeit sinnvoll aufzuteilen (36). Die „Reichsarbeit" sei „glorreich“ durchgeführt worden (36). Allerdings ist die Funktionsfähigkeit des Systems an die Person Diokletians gebunden, dessen überlegene Stellung alle Tetrarchen, so unterschiedlich sie ge-

3 Zur Entwicklung des Diokletiansbildes ist wenig Literatur vorhanden, s. immerhin den Forschungsuberblick in KolB 1987, lff.; als Seitenblick HIGHAM 1997, der aus der Sicht eines Geschäftsmannes schreibt.

4 Zu Burckhardts Diokletians-Bild einige kurze Bemerkungen bei KAEGI 1956, 404f., dessen Bemerkungen zum Werk als Ganzes 377ff. weiterhin unverzichtbar sind; ferner NoLL 1997, 143ff.

5 Ich zitiere Burckhardt hier nach der leichter zugänglichen zweiten Auflage (Leipzig 1880). Die Unterschiede zwischen beiden Ausgaben sind begrenzt: Einiges an Detailforschung aus der jüngeren Literatur wurde eingearbeitet, vor allem aus den Arbeiten von Preuß und Hunziker. Ausdrucklich geändert hat Burckhardt seine Position in Hinblick auf Laktanz' Autorschaft für De mortibus persecutorum (39, Anm. 4); die Historia Augusta spielt eine größere Rolle als Quelle und die Darstellung des Herrschaftssystems wird etwas zugespitzt, doch an der Einschätzung im Grundsätzlichen hält Burckhardt wie auch sonst fest; vgl. zu der geringen Zahl von Änderungen den textkritischen Anhang in der Ausgabe von F. Stähelin, Stuttgart 1929, 373ff. und seine Bemerkungen in der Einleitung XIIf.

6 VOLLMER 1991, insbes. $441 \mathrm{f}$. 
wesen seien, anerkannt hätten (38). Als er auf das Scheitern der Ordnung durch die Usurpation Konstantins zu sprechen kommt, gesteht Burckhardt immerhin den Fehler mangelnder Konsequenz kühl ein: „Man mußte die Kaisersöhne entweder befördern oder hinrichten“ (306). Der Kaiser reagiert auf den Zusammenbruch mit Selbstmord (324f.).

Die Grundlage für die relative Stabilität des Systems ist die Superstition Diokletians selbst und der anderen Tetrarchen: Diokletian glaubt aufgrund von omina an seine Berufung und die übrigen Herrscher auch; sie fügen sich daher entgegen aller politischen und psychologischen Wahrscheinlichkeit seinem Willen (41ff.). Dieses Motiv schlägt Burckhardt gleich im ersten Satz des Abschnittes zu Diokletian an: „Die Vorbedeutungen waren erfüllt und die Orakel hatten Recht behalten, als der Sohn dalmatinischer Sklaven [...] etwa neununddreißigjährig den Thron der Welt bestieg" (35). Die Superstition, die aus der Sicht Burckhardts natürlich eigentlich abzulehnen ist, erweist sich ironischerweise als entscheidendes Hilfsmittel zur Stabilisierung des Reiches - eine typisch Burckhardt'sche Denkfigur, die eine weitere Wendung dadurch erhält, daß Burckhardt den Irrglauben Diokletians, das System werde seinen Rücktritt überdauern, eben auf dessen Superstition zurückführt (305).

Das Lob für das System der Adoptionen steht nicht isoliert. Burckhardt, der die Nachtseiten Konstantins so schonungslos offenlegt, hegt ausgeprägte Sympathien für Diokletian. Es fallen Stichworte wie „imposanter Mensch“ (37) oder „Regentengröße“ (43). Der Kaiser ist ein Mann von einem „hohen, durchdringenden Geist“ (36). Er betrachtet sein Amt als „furchtbar und verantwortungsvoll“, er sieht kein Vergnügen darin (38). Die Schonung der Anhänger des Carinus erklärt Burckhardt nicht durch deren Verrat an ihrem eigentlichen Kaiser, sondern mit der Milde des Kaisers und dessen Wunsch, dem Gemeinwesen zu dienen (32). Die Abschaffung der frumentarii, der institutionalisierten politischen Spionage, sei Ausdruck seiner Großzügigkeit gewesen (55). Kurz, Diokletian ist für Burckhardt eine der bedeutendsten Herrscherpersönlichkeiten überhaupt. Diese Feststellung ist für ihn so unbestreitbar, daß sie wiederholt als Grundlage seiner Argumentation dient. Charakteristisch ist folgende Bemerkung: „Ein Mensch von der Bedeutung und den Erfahrungen Diocletians nimmt die Last einer so gesteigerten Repräsentation (sc. des Hofzeremoniells) nicht ohne genügenden Anlaß auf sich“. ?

Allerdings kann Diokletian auch „sonderbar und räthselhaft" sein (36). Diese Eigenart kommt zum Beispiel in den erwähnten superstitiösen Elementen des Regierungssystems zum Ausdruck. Sie ist Ausdruck der Zeitstimmung, deren religiöses Suchen Burckhardt im sechsten Abschnitt eindringlich schildert, und ist daher, wie gezeigt, gerade nicht dysfunktional. ${ }^{8}$

7 47; vgl. etwa auch 43, 290 f., 300.

8 Diese positive Einschätzung Diokletians hat Burckhardt beibehalten, s. CHRIST 1963, 108. 
Burckhardt vermag nicht zu bestreiten, daß sein Held auch unabhängig von seinem Aberglauben eine Reihe von Angriffspunkten bietet. Das Preisedikt etwa hält auch er für im Ansatz mißlungen, doch verzichtet er nicht einmal hier auf eine Rechtfertigung, indem er es als eine Antwort auf Mißstände im Osten des Reiches interpretiert, wobei er erst in der zweiten Auflage, an diesem Punkte offenbar von der jüngeren Detailforschungen beeinflußt, von Spekulanten spricht (61f.). Ein schwierigeres Problem stellt das Hofzeremoniell dar, das Burckhardt mißbilligt und dem Orient zuordnet $(54,289,306)$. Es sei im Zusammenhang der „Feierlichkeit“ zu sehen, mit der Diokletian sich auch sonst umgeben habe (45). Es dient ferner dazu, ihn und seine Kollegen von den übrigen Menschen, zumal den früheren Freunden, abzusetzen und damit die „Harmonie“ untereinander zu sichern (46); ferner will der Kaiser vermeiden, so erwägt Burckhardt jedenfalls, die Barbaren im römischen Dienst, die den kameradschaftlichen Ton nicht beherrschen, auszuschließen; überdies sollen die Christen am Hof davon abgehalten werden, mit den Heiden Diskussionen zu führen. Im übrigen weiß Diokletian selbst um dessen Nachteile und fühlt sich unbehaglich dabei (47, vgl. 306). Die Ausbreitung des Titel- und Rangwesens schließlich ist nicht Diokletian persönlich anzulasten, sondern Teil eines allgemeineren Entwicklungsprozesses der ausgehenden Antike, und erst Konstantin ist es, der den Staat endgültig umgestaltet. ${ }^{9}$ Erneut wird deutlich, wie sehr Diokletians Wirken bei Burckhardt den Zeitströmungen verhaftet ist.

Den entscheidenden Punkt habe ich bisher ausgespart, und auch Burckhardt umgeht ihn bei der Darstellung der Regierung Diokletians, der in seinen Augen trotz seinem Aberglauben eigentlich tolerant ist: die Christenverfolgung. Erst im achten Abschnitt, viele Seiten später, bevor er vom Ende Diokletians handelt, kommt dieses Ereignis, in der Überschrift hervorgehoben, zur Sprache; ironischerweise schildert Burckhardt in eben diesem Kapitel den Aufstieg Konstantins, der dem Christentum zum Durchbruch verhelfen sollte. ${ }^{10}$

Bedeutungsvoll sind die quellenkritischen Überlegungen, die Burckhardt anstellt: Euseb und Laktanz verwirft er völlig, selbst den dort belegten Einfluß des Galerius bestreitet er. Damit gerät er in eine Aporie. Denn es bleibt, wenn diese beiden Quellen ausfallen, fast nichts an antiken Nachrichten übrig, zumal Burckhardt mit Bedauern feststellen muß, daß Zosimos und Ammian, die er achtet, für die fraglichen Jahre allenfalls spärliche Informationen bieten. In dieser mißlichen Situation formuliert Burckhardt ein bemerkenswertes methodisches Prinzip: „Von da an sind also die Vermuthungen in ihrem Rechte, sobald sie nicht in der Luft schweben, sondern den

9 SIEBERT 1991, 81, 86 scheint mir zu stark diese potentiell negativen Aspekte des Diokletiansbildes zu betonen.

10 GOSSMAN 2000, 268. 
echten vorhandenen Spuren nachgehen und zu dem sonstigen Charakter der Zeit und der handelnden Personen passen" (292).

Und den Charakter Diokletians hat Burckhardt ja schon längst umrissen: Diokletian besitzt eine hohe persönliche Moralität, so daß Grausamkeiten, wie sie eine Christenverfolgung mit sich zu bringen pflegt, ${ }^{1}$ nicht zu ihm passen. Wenn die Maßnahmen erklärlich sein sollen, müssen sie etwas mit dem hohen Verantwortungsbewußtsein des Kaisers zu tun haben.

Zunächst schließt er einige denkbare Motive aus: Weder habe Diokletian einem Ausbruch von Volkswut gegen die Christen oder den Einflüsterungen heidnischer Priester nachgegeben noch sei er von dem Eindruck geleitet gewesen, die Christen gefahrdeten die Moral. Beides sieht der Historiker nicht als belegt an (292f.); die inneren Konflikte unter den Christen wiederum seien weder heftig noch bedrohlich genug für eine solche Intervention gewesen (293f.).

Burckhardt bezieht sich seinerseits auf zwei Quellen, die den Charakter von Überresten haben und dokumentieren sollen, daß Diokletian das Gefühl haben mußte, von Verrat bedroht zu sein. Es handelt sich zum einen um eine Inschrift aus Ascoli, in der davon die Rede ist, daß jemand den Kult der Christen, die versucht hätten, den Staat zu unterminieren, gänzlich vernichtet habe. Sie ist für Burckhardt das Indiz für eine Verschwörung. ${ }^{12}$ Der andere Text ist ein Brief des alexandrinischen Bischofs Theonas an den praepositus sacri cubiculi Lucianus, der von der großen Anzahl der Christen am Hof spricht und diesen Maßregeln mitteilt, um den Glauben zu verbreiten (295). Beide Belege sind mehr als zweifelhaft: Die Inschrift hat man schon lange und sicher zu Recht als falsa klassifiziert, ${ }^{13}$ der Theonas-Brief ist als frühneuzeitliche Fälschung entlarvt. ${ }^{14}$

Im Anschluß an diese Quellendeutungen führt Burckhardt den hohen Anteil von Christen im kaiserlichen Heer (296f.) als weiteren Grund für die Christenverfolgungen an. Diokletian habe das Gefühl gehabt, sich nicht mehr auf den Gehorsam seiner Truppen verlassen zu können, wenn sie christlich gesonnen seien, und habe daher eine Reinigung vorgenommen. Schließlich erwähnt er zwei vermutlich christlich inspirierte Aufstände, auf die Euseb anspielt (hist. eccl. VIII 6,8-10), von denen Burckhardt indes selber weiß, daß sie nicht zuverlässig in die Zeit vor die Christenver-

11 Und immerhin bestreitet Burckhardt nicht, daß es solche gegeben habe, s. S. 137.

12 294f. Es handelt sich um CIL IX 525*: [...] Christianorum cultu qui rem publicam sub. vertebant, prorsus deleto [...].

13 So auch im CIL, wie die Numerierung zeigt. Burckhardt erwăhnt 294, Anm. 2, daß L. A. Muratori als Herausgeber die Inschrift unter die Falschen eingeordnet habe, argumentiert aber nicht dagegen, sondern erklärt lediglich mit seinem charakteristischen Desinteresse an Detailarbeit, daß die anderen dort angegebenen Inschriften verdächtiger seien.

14 S. nur HARNACK 1903, der im Anschluß an P. Batiffol den Text als eine Fälschung aus der Zeit Ludwigs XIV. (1643-1715) erweist. 
folgungen zu datieren sind. In der zweiten Auflage geht er fast so weit, einzuräumen, daß die Unruhen erst nach dem Beginn der Verfolgungen ausgebrochen seien, wischt das Eingeständnis aber vom Tisch: „Aber auch dies setzt wahrlich eine schon vorher sehr bedenkliche Stimmung voraus" (297, Anm. 1). Ferner versucht Burckhardt, das harte Vorgehen gegen christliche Palastbeamte, die zu Märtyrern wurden, sowie die Palastbrände in Nikomedien mit einer christlichen Verschwörung in Zusammenhang zu bringen (299f.). Aus den Bestrebungen einzelner habe der Kaiser unglücklicherweise einen allgemeinen Verdacht gegen die Christen abgeleitet (301).

Es sind außerordentlich schwache Indizien, die Burckhardt zusammenträgt; insgesamt aber entsteht die Suggestion, daß staatspolitische Verantwortung Diokletian zu seinem Handeln getrieben habe, die Sorge wegen einer Verschwörung, die von Christen in seinem Umkreis ausgegangen sei. Unter den vielen apologetischen Urteilen Burckhardts über Diokletian ist das zu den Christenverfolgungen das krasseste. Die Kritik daran wurde sogleich nach dem Erscheinen des Buches laut. ${ }^{15}$ Dennoch verzichtete Burckhardt nicht darauf, seine Argumentation auch in der zweiten Auflage zu wiederholen.

Will man sich vor einer vordergründigen Sicht auf die Bewertung des Kaisers durch den Baseler hüten, muß man noch einen Schritt zurück tun. Das gesamte Werk ist geprägt von einem organizistischen Geschichtsmodell, das weit mehr darstellt als eine Metapher: Das Römische Reich altert und ist mithin zur Erstarrung verdammt. ${ }^{16}$ Danach bemißt sich, wie schon an einzelnen Punkten deutlich geworden ist, der Handlungsspielraum der Akteure, sogar der einer herausragenden Herrschergestalt wie Diokletian: „Das Höchste, was selbst ein Regent wie Diocletian zu erreichen hoffen durfte, war die Erhaltung des Reiches in seinem Umfang und eine leidliche Ausbesserung der Schäden im Innern" (66).

Und noch mehr steckt darin. Dies wird deutlich, als Burckhardt von der Lösung des Reiches von seinen italischen Traditionen durch Diokletian spricht: Wenn er das nicht getan hätte, ,so hätte doch bald darauf das Christenthum dieselbe Aufgabe auf seine Weise vollbringen müssen, indem es mit Nothwendigkeit ganz neue Schwerpunkte der Macht schuf" (54). Gegen die Eigendynamik des historischen Verlaufs ist der Handelnde machtlos, ein Grundmotiv des Burckhardt'schen Denkens. ${ }^{17}$ Bezeichnend ist die Wirkung der Christenverfolgung: Sie stärkt letztlich die Verfolgten. Man sehe sie „durch den Untergang siegen“" (303).

So ist die Geschichte Diokletians bei Burckhardt die Geschichte der Selbstbehauptung eines fähigen Individuums in unglückseligen Zeiten, einer Gestalt also, mit der Burckhardt, der sich selbst als unzeitgemäß erlebte, besonders leicht Identifika-

15 S. KAEGI $1956,417,419 f$.

16 Vgl. Flaig 1984.

17 Vgl. Gossman 2000, 203ff.; Noll 1997, insbes. 137ff. 
tionsmöglichkeiten fand, einer jener Menschen bei Burckhardt, deren Porträt aus der ironischen Haltung des Historikers erwächst, wie Hayden White sie beschrieben hat. ${ }^{18}$

Burckhardts Darstellung war, wie erwähnt, diejenige, von der die späteren Forscher ausgingen und mit der sie sich auseinandersetzten, da er die Konzeption des diokletianischen Herrschaftssystems aufgezeigt zu haben schien. Wenden wir uns, nicht einer chronologischen, sondern einer systematischen Gliederung folgend, Theodor Mommsen zu. Er hat anders als Burckhardt intensive Detailforschung zum Zeitalter betrieben. So hat er über die Chronologie der Regierung gehandelt und die Rolle der Gesetze sowie aufgrund harter epigraphischer Arbeit die Einzelheiten des Höchstpreisediktes erörtert. ${ }^{19}$ Seine Vorstellung von Diokletian läßt sich indes vor allem aus seinen Kaiserzeitvorlesungen gewinnen, die in den achtziger Jahren gehalten wurden, vor allem aus jenen des Wintersemesters $1885 / 6$ und Sommersemesters $1886 ;{ }^{20}$ hinzu kommen wenige Andeutungen der anderen Schriften, in denen aber kaum prononcierte Wertungen vorkommen. ${ }^{21}$ Bekanntlich sind die Vorlesungen mit Vorsicht zu benutzen, da sie uns heute nur in einer mehrfach gebrochenen Form vorliegen, ${ }^{22}$ wobei es allerdings kaum wahrscheinlich ist, daß die Tendenz der Äußerungen Mommsens insgesamt verfälscht wäre. Andererseits sollte man natürlich auch unter diesen Voraussetzungen an eine Vorlesung nicht diejenigen Erwartungen in Hinblick auf Konsistenz und Systematik herantragen, die man an ein zur Publikation bestimmtes Werk füglich stellen darf.

18 WHITE 1991, 306ff.

19 Etwa: Uber das Edict Diocletians de pretiis rerum venalium (1851), GS, Abt. 1,2, Berlin 1905, 292-322; Das diocletianische Edikt über die Warenpreise (1890), GS, Abt. 1,2, Berlin 1905, 323-340; Über die Zeitfolge der Verordnungen Diocletians und seiner Mitregenten (1860), GS, Abt. 1,2, Berlin 1905, 195-291.

20 Theodor Mommsen, Römische Kaiserzeit. Nach den Vorlesungs-Mitschriften von Sebastian und Paul Hensel 1882/86 hg. von Barbara und Alexander Demandt, München 1992.

21 Theodor Mommsen, Römisches Staatsrecht, 3 Bde., ND Darmstadt 1971, II 1169 setzt etwa ein diokletianisches „System" voraus. Für den nachgeschobenen, die Provinzen behandelnden Fünften Band der „Römischen Geschichte“ (hier zitiert nach der Ausgabe von Karl Christ in 8 Bänden, München 1976) bildet die Epoche Diokletians den zeitlichen Endpunkt. Viel zu seiner Bewertung findet sich nicht. Immerhin wird er einmal als „,Regenerator des römischen Staates" bezeichnet $(6,187)$; eine gewisse Rolle spielt im Rahmen der Provinzialgeschichte lediglich Diokletians Wunsch, Rom die Hauptstadtfunktion zu nehmen, die „Dekapitalisierung Roms“ $(6,187 ; 7,268)$.

22 Zum Problem die Rez. von Karl Christ, GGA 245 (1993), 201-236, 209; 230f. zum Diokletiansbild. 
Mommsen sieht wie Burckhardt in Diokletian einen Kaiser der Verfallszeit (429); einmal gebraucht er eine organizistische Metapher: Unter Diokletian habe eine Verjüngung stattgefunden wie unter Vespasian und Justinian (228). Allerdings ist das organizistische Bild bei Mommsen eben nicht mehr als eine Metapher und anders als bei dem Schweizer kein Modell zur Deutung des Niedergangs. Ansonsten bleiben seine Erklärungen der Wirkung Diokletians nüchtern. Mit seinem Abgang sei das Reich aus den Fugen geraten (474), nur mit Diokletian habe die Tetrarchie funktioniert (505).

Für den Berliner ist Diokletian, nicht Konstantin der eigentliche Begründer der Spätantike. Obwohl er sich bewußt ist, welche Schwierigkeiten es bereitet, den jeweiligen Anteil von Diokletian und Konstantin an den einzelnen Reformmaßnahmen zu bestimmen, fält er ein klares Urteil über den Rang der beiden. „Diocletian bleibt, wenn man ihn noch so genau, noch so kritisch betrachtet, eine großartige, sympathische Persönlichkeit, die Ungeheures geschaffen hat, durch bewußte Geistestat, nicht durch Zufall. Constantin verhält sich zu ihm höchstens wie Augustus zu Caesar; alle genetischen Gedanken sind Diocletians, und das diocletianische, nicht das constantinische müßte das Zeitalter heißen. ${ }^{\text {(23 }}$ Das ist zweifellos auch eine Polemik gegen Jacob Burckhardt und dessen Formel von der Zeit Konstantins des Großen.

In der günstigen Beurteilung der Persönlichkeit Diokletians indes treffen sich die beiden Gelehrten, wenn nicht das Bild, das Mommsen zeichnet, noch enthusiastischer ist als jenes von Burckhardt. Von Genialität spricht der Berliner bei Diokletian, zumal dem Kaiser seine Schwächen bewußt gewesen seien, die vor allem im militärischen Bereich gelegen hätten (473f.); ferner ist die Rede von einer ,einzigartigen Nüchternheit und Klarheit" (474), er sei nicht eitel gewesen (475), dafür eine ehrliche Natur (515), er habe nach der Macht nicht gestrebt (472f.) und eine Neigung zu Milde besessen (474), ja Mommsen erhebt sich sogar zu einem Vergleich mit einer Person, die ihm teuer ist: Diokletian habe einen friderizianischen ökonomischen Sinn besessen (475). ${ }^{24}$ Das Hofzeremoniell, von dem Burckhardt eingehend gehandelt hatte, übergeht Mommsen weitgehend.

Als schwerer wiegende Schwäche erkennt der Berliner lediglich eine gewisse Furcht vor Entscheidungen und Leisetreterei (500). Natürlich mißbilligt er auch einzelne Maßnahmen wie das Preisedikt, das er als undurchführbar bewertet, da es gegen die Natur der Dinge gerichtet sei (495f.), oder den Hang des Kaisers zum Thesaurieren, aber das stört den glänzenden Gesamteindruck - erstaunlicherweise - nicht.

Mommsen sieht bei Diokletian das Bestreben am Werk, das Alte zu erneuern, und betrachtet ihn als einen tiefreligiösen Menschen, dessen besondere Verehrung Sol und Mithras gegolten habe, dem der Monotheismus aber nicht fremd gewesen sei. Doch

23514 ; vgl. 424.

24 Vgl. zur Hochschätzung Friedrichs des Großen durch Mommsen ReBENICH 2002, 188 
fehle jeglicher Fanatismus (475). Allerdings macht Mommsen die religiöse Haltung nicht zum Movens der Politik, da Diokletian seiner Auffassung nach eigentlich tolerant war (499), was ihn wieder mit Friedrich dem Großen verbindet, ohne daß Mommsen dies ausfuihrt.

Selbst das Manichäeredikt erklärt der Berliner ausdrücklich mit politischen, nicht mit religiösen Motiven. Was aber kann er aus den Christenverfolgungen machen? Ein grundlegender Unterschied zu Burckhardt liegt darin, daß Mommsen die mehr als zweifelhaften Zeugnisse, die Burckhardt zum Beleg einer christlichen Verschwörung anführt, schlicht ignoriert. An der entsprechenden Stelle findet sich sogar, was sehr selten ist, eine unmittelbare Polemik gegen Burckhardt. Die ,großen Ursachen konnte Lactantius nicht sehen, wie sie sehr oft ein Zeitgenosse nicht sehen kann. Burckhardt aber konnte es noch weniger sehen" (497).

Und dann folgt eine Konfrontation zwischen Heidentum und Christentum, die bezeichnend ist für die notorische religiöse Unmusikalität des Pfarrerssohnes: „Das Prinzip der römischen Staatsreligion ist mit einem Wort ausgedrückt: Toleranz" (497). Sie verkörpert somit einen der höchsten politischen Werte für Mommsen. Welche Wirkungen diese Toleranz zeitigt, beschreibt er ausfuihrlich, um zu folgendem Ausruf überzuleiten: „Aber anders (also nicht tolerant) die Juden und die Christen“ (498). Das Christentum war unduldsam, zerstörte die alten Götter und die alte Bildung, es bildete einen Staat im Staate. „Faßt man alle diese Momente ins Auge, so wäre man beinah versucht zu sagen, die Christenverfolgungen seien entschuldigt. Der Staat mußte sich gegen Proselytismus, gegen Hierarchie, gegen alle Prinzipien des Christentums wehren. Es ist ein höchst merkwürdiger Umstand, daß gerade die schwächsten Kaiser dem Christentum am meisten die Zügel schießen ließen“ (499). Genau genommen entschuldigt Mommsen die Verfolgungen tatsächlich: Diokletian handelt gewissermaßen im Staatsnotstand, auch wenn Mommsen dann in engem Anschluß an Laktanz den Anlaß von religiösen Ereignissen ableitet (500) und Galerius als eigentlichen Motor der Entwicklung bezeichnet (500f.). Wenn somit eine Entlastung stattfindet, bleibt das Bild Diokletians frei von ernstlichem Makel. Die antikatholische Stimmung des Kulturkampfes, der tobte, als die Vorlesungen gehalten wurden, mag bei Mommsen nachgeklungen haben.

Damit zu zwei - mit Recht - weniger bekannten Forschern, die allerdings deswegen von wissenschaftsgeschichtlichem Interesse sind, weil ihre Werke dezidiert christliche Versuche bilden, zu einer adäquaten Einschätzung Diokletians zu gelangen. Es handelt sich um Albrecht Vogel und Theodor Preuß. 
Albrecht Vogel (1822-1890), außerordentlicher Professor an der liberalen theologischen Fakultät zu Jena, ${ }^{25}$ hielt für eine Angehörige der großherzoglichen Familie eine Rede über Diokletian, die er 1857 um ausführliche Anmerkungen ergänzt herausgab. ${ }^{26}$ Vogel schreibt bewußt als christlicher Historiker und Theologe; als solcher setzt er dazu an, einer christlichen, und das heißt für ihn, einer einseitig negativen Interpretation des Kaisers entgegenzutreten, unter prononciertem Anschluß an Jacob Burckhardt. Dies geschieht in einer narrativ eingängigen, eben auch für eine Prinzessin faßbaren Form und ohne Scheu vor psychologischen Spekulationen.

Diokletian wird wegen seiner Weisheit zum Kaiser gewählt, die sich in dem Aufbau eines wunderbar „künstlichen“ - hier im Sinne des modernen Adjektivs „,kunstvoll" gebraucht - Systems niederschlägt (15), an dem indes die hohe Steuerlast dem Redner nicht behagt (18ff.). Auch kriegerische Erfolge erzielt Diokletian in großer Zahl (32ff.). Doch Tadel verdienen die religiösen Pläne Diokletians für das Heidentum. Dazu erklärt Vogel: „Da zeigte sich doch nur eine hitzige, pedantische Religiosität in der Form einer einzig berechtigten und unumschränkt herrschenden Staatskirche“ (22).

Diesem Phänomen stellt der Kirchenhistoriker die Entwicklung des Christentums gegenüber. Die ursprünglich orientalische Religion sei „zu einer Christenheit geworden, welche das römische Reich völlig zu ersetzen bestimmt war" (27). Ja, sie „war zum Träger des monarchischen Gesamtstaates herangewachsen und sehnte sich, den Schritt zur Herrschaft durch Christianisierung des Staatsoberhauptes zu thun" (28f.). Hier kämpft also eine geschichtliche Kraft, das Christentum, gegen eine künstliche Größe, und der Sieger steht von vornherein fest. Auch Diokletian erkennt nach seinen vielen militärischen Siegen, was kommt. Er sieht „schwermütig“ (40), so weiß Vogel, einen Kampf zwischen Christentum und Heidentum voraus, spürt aber, daß Verfolgungen „Frevel“ wären. Und dann schwingt Vogel sich rhetorisch in die Höhe: „Sein Frevel war in Galerius persönlich geworden" (41). Dieser wird zum eigentlich Schuldigen, indem er Diokletian zu den Verfolgungen drängt, deren Entstehung und Anlaß der Kirchenhistoriker in engem Anschluß an Laktanz erzählt und, ohne daß er von Burckhardt die These übernimmt, Diokletian habe einen Verrat seitens der Christen gefürchtet. Neu ist, daß die Christenverfolgungen der Grund für den Rücktritt Diokletians gewesen sein sollen: „Ihn schauderte vor den blutigen Geleisen, aus welchen sein kaiserlicher Triumphwagen nicht mehr herauszubringen war" (45). Auch damit will er offenbar das Bild des Christenverfolgers bannen.

Soweit zu den Einzelheiten von Vogels Revision des christlichen Diokletiansbildes. In der Tat ist das Porträt, das er entwirft, über weite Strecken positiv. Er betrachtet

$25 \mathrm{Zu}$ ihm HeussI 1954, 263.

26 Albrecht Vogel, Der Kaiser Diokletian. Ein Vortrag am 10. Dezember 1856 zu Jena gehalten und mit Anmerkungen herausgegeben, Gotha 1857. 
Diokletian als verantwortungsvollen Kaiser und spricht ihm sogar die Fähigkeit zu, einen eigenen Fehler einzusehen. Mit Jacob Burckhardt gemeinsam hat der Jenaer, daß er im Sieg des Christentums eine notwendige Entwicklung sieht. Doch was bei Burckhardt Ausdruck des Alterns ist und dazu dient, mühsam die Reste der alten Welt zu bewahren, eröffnet in Vogels frommer Perspektive eine neue Welt. Denn das Christentum ist anders als bei Mommsen oder Burckhardt keine Kraft, die die bestehende Ordnung sprengt, sondern ein Träger des Staates, der heimlich selbst zum Telos der Geschichte zu werden scheint.

Damit zu Theodor Preuß, dem Oberlehrer am Insterburger Gymnasium in OstpreuBen. Von ihm stammt die maßgebliche, zum Nachschlagen geeignete, systematisch mit Quellenbelegen versehene Darstellung des Zeitalters Diokletians aus dem 19. Jahrhundert. Sie erschien 1869, also nach Burckhardts erster Auflage, aber bevor Mommsen seine Vorlesungen hielt. ${ }^{27}$ Viele Jahrzehnte blieb sie das wichtigste Standardwerk.

Das klar geschriebene Buch bietet gleich auf der ersten Seite ein weltanschauliches Bekenntnis. Das Römische Reich war eine Wohltat für die Völker, vor allem weil es die Ausbreitung des Christentums erlaubt habe, erklärt Preuß mit fast eusebianischer Geisteshaltung. Wer aber nun erwartet, einem rabiaten Polemiker gegen den Christenverfolger Diokletian zu begegnen, der sieht sich getäuscht. Wie Burckhardt so handelt auch Preuß ausführlich über die Erfolge Diokletians bei der Sicherung und Neuordnung des Reiches. Allerdings setzt er sich, wie auch gar nicht anders möglich, von Burckhardt ab, als es um die Ursachen der Christenverfolgungen geht. Dessen Verschwörungstheorie lehnt er ab (136, 139, Anm. 1). Er rekurriert vielmehr auf welthistorische Notwendigkeiten. Wie später Mommsen sieht er in der Kirche einen Staat in und neben dem weltlichen Staat (141), doch ist sie bei ihm gerechtfertigt, da sie die historische Mission hat, das Römische Reich weiterzuführen. Wenn Diokletian daher gegen die Christen kämpft, so begeht er einen historischen Fehler. Nicht moralisch wird argumentiert, nicht mit der Idee der Toleranz, sondern mit einem letztlich heilsgeschichtlichen Konzept. So kann auch das Folgende nicht ausbleiben: „In demselben Jahre, da jenes Edict erschien, verlassen ihn (sc. Diokletian) Kraft und Glück“ (171). Der Kaiser erlebt also eine unmittelbare göttliche Strafe. Dieses Geschichtsbild kommt noch einmal in den pathetischen Worten des Abschlusses zum Ausdruck: „Der kluge Fürst, der in staatlicher Hinsicht die Forderungen seiner Zeit so klar erfasste die tiefere Bewegung, die religiöse Strömung seiner Zeit hat er nicht verstanden. Das ist seine geschichtliche Verschuldung und er hat sie gebüsst. Er und sein Haus wurden verworfen und ein anderer erwählt, sein Werk zu vollenden." Die Geschichte läuft auf

27 Theodor Preuß, Kaiser Diocletian und seine Zeit, Leipzig 1869. 
Konstantin zu. Noch viel stärker als von Vogel wird somit von Preuß mit religiösen Kategorien gearbeitet, auch wenn er den Namen Gottes nicht mehr im Munde führt. Auch die Heilsgeschichte steht im Schatten der Säkularisierung.

Diokletian ist, das zeigt sich, wenn man auf das Gesagte zurückblickt, mit aller Deutlichkeit, ein stiller, aber erstaunlich breit anerkannter Held des 19. Jahrhunderts. Sein Charakterbild schwankt weniger in der Geschichte, als man erwarten sollte; vieler Parteien Gunst fallt ihm zu. Inmitten einer schlechten Zeit wirkt er als Lichtgestalt. Alle Autoren loben Diokletians außenpolitischen Erfolge, alle heben die Stabilität der Tetrarchie, die sie als System begreifen, hervor, solange er im Amt blieb. Auch über die Fehler besteht weithin Konsens: das Preisedikt, die übergroße Schematisierung des Herrschaftssystems werden kritisiert; doch können sie der positiven Grundeinschätzung offenbar nichts anhaben.

Obgleich bei den Diskussionen um Diokletian stets auch von Konstantin die Rede war, ist die Hochschätzung Diokletians keineswegs an die Bewertung Konstantins des Großen gekoppelt: Dessen Bewunderer und Verächter gelangen gleichermaßen zu einem wohlwollenden Urteil über Diokletian; dies erweisen die beiden christlichen Autoren, die anders als Burckhardt und Mommsen Konstantin große Verehrung entgegenbringen. Zweierlei dürfte dahinter stehen. Zum einen kommt die Darstellung eines einzelnen, der sich zumal im Wissen um die Vergeblichkeit seines Bemühens einer Entwicklung entgegenstemmt, dem bildungsbürgerlichen, oft als tragisch bezeichneten Selbstverständnis entgegen, das sich besonders in der 2. Hälfte des 19. Jahrhunderts unter dem Eindruck der industrialisierten Massengesellschaft herausbildete; zum anderen ist die Ordnung eines Staatswesens nach außen und nach innen und die entsprechenden Erfolge Diokletians sind schwerlich zu bestreiten - im 19. Jahrhundert natürlich von zentraler Bedeutung für die Bewertung von Herrschern. Nicht primär als religiöse Gestalt wird Diokletian somit im 19. Jahrhundert wahrgenommen, vielmehr dient seine Geschichte als Projektionsfläche bürgerlicher Staatsvorstellungen.

Auch wenn bei der Erörterung der höfischen Strukturen die Frage nach dem persischen Einfluß eine gewisse Rolle spielt, hat die Vorstellung einer Orientalisierung keine zentrale Bedeutung, wie auch der illyrischen Herkunft des Tetrarchen kein großes Gewicht beigemessen wird. Der Ethnizismus hat die Geschichtswissenschaft noch nicht im Griff.

Bemerkenswert ist, daß die beiden hier behandelten dezidiert christlichen Historiker sich der günstigen Bewertung Diokletians nicht entziehen wollen und können: Die Hegemonie säkularer Kategorien prägt auch diese Autoren. Selbst in Hinblick auf die Christenverfolgungen geht der Konsens erstaunlich weit. Einig ist man sich etwa 
darin, daß das Christentum am Ende den Sieg davontragen müsse, so daß der Kampf gegen diese Religion notwendig scheiterte. Keinem der vier passen jedoch die Verfolgungen so recht ins Konzept, weder Jacob Burckhardt und Theodor Mommsen, die Diokletian als milde oder gar tolerant betrachten und seine Weitsicht rühmen, noch Albrecht Vogel und Theodor Preuß, die ihn als einen tüchtigen Herrscher sehen. Einig ist man sich daher noch in dem Bestreben, Diokletian zu entlasten und, mit der Ausnahme Burckhardts, die Hauptschuld Galerius zuzuschreiben. Doch hier setzen entscheidende Unterschiede an.

Burckhardt muß sich mit einer sehr persönlichen Art der Quellenverwertung aushelfen, um einen Verrat von Christen zu belegen und die Maßnahmen Diokletians zu legitimieren; Vogel gelangt zu der Annahme, daß Diokletian wider Willen die Christenverfolgung eingeleitet habe, während Preuß und Mommsen eine im Grundsätzlichen gemeinsame Position beziehen. Für beide hat das Christentum eine erhebliche eigene Macht gewonnen und stellt daher eine Gefahr für das diokletianische Regierungssystem dar. Es ist bezeichnend für den liberalen Preußen Mommsen und eben auch für seine Neigung zur Übertragung von Phänomenen seiner Zeit auf die Vergangenheit, daß er daraus eine Rechtfertigung der Christenverfolgungen ableitet und eine letztlich ahistorische Kategorie der Toleranz verwendet, während der fromme Preuß in den Maßnahmen einen Fehler entdeckt, ein Handeln gegen Gottes Willen, und die Strafe alsbald folgen läßt.

An den deutlich erkennbaren Schwierigkeiten aller Autoren, die Christenverfolgungen mit ihrem jeweiligen Bild Diokletians in Einklang zu bringen, zeigt sich, daß bei aller Dominanz säkularer Kategorien das Christentum mangelhaft kontextualisiert wird. Dadurch, daß es selbst bei Mommsen und Burckhardt als eine historische Größe mit eigengesetzlicher Entwicklung erscheint, gelingt es nicht, seine Geschichte in das historische Gesamtbild zu integrieren.

Doch zurück zur vorhin gemachten Beobachtung. Die Einmütigkeit in den meisten Punkten ist bemerkenswert. So etwas aber ist verdächtig, und bezeichnenderweise wird auch bald eine Stimme laut, die ein ganz anderes Urteil fält, nämlich jene Otto Seecks. ${ }^{28}$ Seine „Geschichte des Untergangs der antiken Welt" beginnt mit der Abdankung Diokletians und Maximians, und der Autor gibt alsbald im Rahmen eines Rückblicks eine Charakteristik Diokletians. Dessen Leistung als Friedensbringer für das Reich erkennt Seeck durchaus an, auch wenn er seine Persönlichkeit skeptisch sieht: „Diocletian [...] war ein wunderlicher Ideologe, ein grüblerisches Halbgenie, reich an Einfällen, aber arm an Menschenkenntnis und praktischer Lebensweisheit, einer von jener Art, aus der heutzutage die gewerbsmässigen Erfinder hervorgehn" (lf.). Er sei eher als Berater denn als Kaiser geeignet gewesen. „Rohheit des Rechts-

28 Otto Seeck, Geschichte des Untergangs der antiken Welt, 6 Bde., Berlin 1920-23 ${ }^{2-4}$ (ND Darmstadt 2000). Zu ihm REBENICH 1998 und REBENICH 2000. 
gefühls" (4) sei für seine ganze Regierungszeit charakteristisch, das Höchstpreisedikt begreift der Historiker als einen „thörichten Versuch“ (5), von der „Rücksichtslosigkeit“ (in einem allerdings nicht unbedingt negativen Sinne) seiner Reformen ist die Rede, seine Schöpfungen seien „baufällig“ (8). Als gewerbsmäßiger Soldat ist Diokletian diszipliniert, aber auch ängstlich; militärische Begabung fehlt ihm, aber das weiß er auch (22f.) - dies hatte Seeck vielleicht bei Mommsen gelernt. Die Erhebung Maximians zum Mitaugustus wird Diokletian gleichsam aufgezwungen (26). Kurz, der Kaiser ist bei Seeck ein vollkommener Repräsentant seiner Epoche (9) - eine Einschätzung, die den Historiker von seinen Vorgängern grundlegend unterscheidet. ${ }^{29}$

Wo bleiben die Christenverfolgungen? Das fragt man sich immer wieder, wenn man die entsprechenden Passagen bei Seeck durchgeht, und sie fehlen tatsächlich weitgehend, nur kurz wird erwähnt, daß sie blutig waren und ihr Ziel verfehlten (36); erst im IV. Hauptteil (im dritten Band des Gesamtwerks), der über Religion und Sittlichkeit handelt, kommt Seeck noch einmal auf diese Vorgänge zu sprechen und leitet sie aus religiösen Motiven ab, vor allem aber aus dem Verdruß des Kaisers über die Geldgier seiner Untertanen, der er sich ja auch in der Einleitung zum Preisedikt Luft macht: Die Christen haben gleichsam eine Sündenbockfunktion. Im Unterschied zu Galerius konzediert Seeck Diokletian eine gewisse Zurückhaltung, um dann die Verfolgungen in einer an Laktanz angelehnten Drastik zu erzählen. ${ }^{30}$ Diese geringe Gewichtung ist nicht so abwegig, wie es erscheinen mag, denn bei dem Greifswalder Historiker passen die Verfolgungen ins Bild, da seinem Diokletian die Grausamkeit und Unfähigkeit eignet, die für ihn das Signum der Epoche sind, zumal nach Seecks Auffassung sich niemand der Prägung durch seine eigene Epoche entziehen kann. ${ }^{31}$

Während in den Augen der übrigen hier behandelten Forscher Diokletian in einem bemerkenswerten Maße unter seinen Zeitgenossen herausragt, ist er bei Seeck ein führender Repräsentant seines Zeitalters. Zwar verweisen auch die anderen Historiker auf die Grenzen der Handlungsmöglichkeiten eines Diokletian, doch steht er als Individuum gleichsam über oder wenigstens außerhalb seiner Zeit. Bei Seeck hingegen wird die ganze Erbärmlichkeit und Grausamkeit der Epoche auch in diesem Kaiser sichtbar.

Die Bewertung Diokletians, das läßt sich als Ergebnis dieser gerafften Studie festhalten, ist nicht determiniert von den religiösen Positionen der Autoren; vielmehr herrscht, sieht man von Otto Seeck ab, ein sehr vorteilhaftes Bild Diokletians vor, sowohl bei explizit christlichen, als auch bei säkular orientierten Historikern und unabhängig davon, welches Urteil sie über Konstantin fällen. Prägend für das Bild aller

29 Das hindert ihn nicht, positiv auf Burckhardt Bezug zu nehmen, vgl. etwa I $437 \mathrm{zu} 3,3$ (zur Tötung Apers) und I 463 zu 36,24 (zum System der Tetrarchie).

30 III 307ff. 308f. motiviert er das Manichäeredikt wie Mommsen politisch.

31 Vgl. zu Seecks Geschichtsbild LePPIN 1998. 
war, was Jacob Burckhardt beschrieben hatte und was in den achtziger Jahre in den eigentlich unangemessenen Begriff der Tetrarchie gerann. Die Schöpfung einer neuen Staatsordnung wird selbst bei dem kritischen Seeck als eine Leistung Diokletians angesehen, und das wog in diesem Jahrhundert offenbar schwerer als mögliche religiöse Vorbehalte.

\section{Bibliographie}

CHRIST, KARL, Jacob Burckhardt und die Römische Geschichte, Saeculum 14, 1963, 82-122. [= CHRIST 1963]

FlAIG, EGoN, Der Begriff 'Alterung' in Jacob Burckhardts 'Zeit Konstantins des Großen', Archiv für Begriffsgeschichte 28, 1984, 201-213. [= FLAIG 1984]

Gossman, Lionel, Basel in the Age of Burckhardt. A Study in Unreasonable Ideas, Chicago/London 2000. [ = Gossman 2000]

HARNACK, ADOLF, Der gefalschte Brief des Bischofs Theonas an den Oberkammerherm Lucian, Texte und Untersuchungen zur Geschichte der altchristlichen Literatur 24,3 $=$ N. F. 9,3, Leipzig 1903. [= HARNACK 1903]

HeussI, KARL, Geschichte der Theologischen Fakultät zu Jena, Weimar 1954. [= Heussi 1954] HIGHAM, ADRIAN, In Search of Diocletian, Classics Ireland 4, 1997, 57-66. [= HIGHAM 1997]

HUNZIKER, OTTO, Zur Regierung und Christenverfolgung des Kaisers Diocletianus und seiner Nachfolger, in: Büdinger, Max ( $\mathrm{Hg}$.), Untersuchungen zur röm. Kaisergeschichte, 3 Bde., Leipzig 1868 (ND Graz 1973), II 116-286. [= HuNZIKER 1868]

KAEGI, WERNER, Jacob Burckhardt. Eine Biographie III, Basel/Stuttgart 1956. [= KAEGI 1956]

KolB, FrANK, Diocletian und die Erste Tetrarchie. Improvisation oder Experiment in der Organisation monarchischer Herrschaft?, Berlin 1987. [= KOLB 1987]

LEPPIN, HARTMUT, Ein „Spätling der Aufklärung“. Otto Seeck und der Untergang der antiken Welt, in: Losemann, Volker/Kneißl, Peter (Hgg.), Imperium Romanum. Studien zu Geschichte und Rezeption. Festschrift für Karl Christ zum 75. Geburtstag, Stuttgart 1998, 472-491. [= LEPPIN 1998]

MADER, FRANZ, Versuch einer Darstellung des Zeitalters des Diokletian, Garms 1847. [= MADER 1847]

Noll, THOMAs, Vom Glück des Gelehrten. Versuch über Jacob Burckhardt, Göttingen 1997. [= NoLl 1997]

Rebenich, Stefan, Otto Seeck, Theodor Mommsen und die „Römische Geschichte“, in: Losemann, Volker/Kneißl, Peter (Hgg.), Imperium Romanum. Studien zu Geschichte und Rezeption. Festschrift für Karl Christ zum 75. Geburtstag, Stuttgart 1998, 582-607. [= REBENCH 1998]

REBENICH, Stefan, Otto Seeck und die Notwendigkeit, Alte Geschichte zu lehren, in: Calder III, William u. a. (Hgg.), Wilamowitz in Greifswald. Akten der Tagung zum 150. Geburts- 
tag Ulrich von Wilamowitz-Moellendorffs in Greifswald, 19.-22. Dezember 1998, Hildesheim/Zürich/New York 2000, 262-298. [= REBENICH 2000]

Rebenich, Stefan, Theodor Mommsen. Eine Biographie, München 2002. [= ReBenICH 2002]

SCHLOSSER, FRIEDRICH CHR., Weltgeschichte für das deutsche Volk, bearb. von G. L. Kriegk,

4. Bd., Frankfurt a. M. 1846. [= Schlosser 1846]

SIEBERT, IRMGARD, Jacob Burckhardt. Studien zur Kunst- und Kulturgeschichtsschreibung, Basel 1991. [= SIEBERT 1991]

VOLLMER, DANKWARD, Tetrarchia. Bemerkungen zum Gebrauch eines antiken und modernen Begriffes, Hermes 119, 1991, 435-449. [= VolLMER 1991]

WHTE, HAYDEN, Metahistory. Die historische Einbildungskraft im 19. Jahrhundert in Europa, Frankfurt a.M. 1991 (engl. 1973). [= WHITE 1991] 